\title{
EZ: An Easy Way to Conduct a More Fine-Grained Analysis of Faked and Nonfaked Implicit Association Test (IAT) Data
}

\author{
Jessica Röhner ${ }^{\mathrm{a}, \bigotimes}$ and Philipp Thoss ${ }^{\mathrm{a}}$ \\ ${ }^{\mathrm{a} C h e m n i t z ~ U n i v e r s i t y ~ o f ~ T e c h n o l o g y ~}$
}

\begin{abstract}
Although faking on the Implicit Association Test (IAT) is a relevant problem, it has not yet been considered for the traditional IAT effect ( $D$ measure). Research has suggested that diffusionmodel-based IAT effects may be useful as $I A T_{v}$ is related to the construct-related variance and $I A T_{\mathrm{a}}$ and $I A T_{t_{0}}$ have both been assumed to provide indications of faking. Recent research used fast-dm to reanalyze nonfaked and faked IAT data under various faking conditions (faking low vs. faking high scores in a naïve vs. informed manner). The results showed that faking affected $I A T_{v}$. However, there was an impact on $I A T_{\mathrm{a}}$ when people knew how to fake and had to fake low scores. Thus, diffusion model analyses deliver additional information, but they are also very complex to perform. The diffusion tool EZ is easy to handle and very powerful, but researchers do not yet know whether $I A T_{v}, I A T_{\mathrm{a}}$, and $I A T_{t_{0}}$ deliver similar information about the components in IAT results when they are obtained with EZ. Thus, we used EZ to reanalyze the data set described above. The results from fast-dm and EZ were comparable, but EZ had somewhat higher statistical power. $I A T_{v}$ was impacted by faking, thus replicating the finding that diffusion model analyses cannot yet be used to completely separate construct- and faking-specific variance from each other. However, replicating and extending the findings that were obtained with fast-dm, informed faking had an impact on $I A T_{\mathrm{a}}$ and $I A T_{t_{0}}$, which might both serve as indicators of faking. Thus, our results indicate that $\mathrm{EZ}$ as well as fast-dm is a powerful tool that can help researchers to interpret IAT results.
\end{abstract}

Keywords — Implicit Association Test (IAT), diffusion model analyses, IAT effects, fast-dm, EZ.

jessica.roehner@psychologie.tu-chemnitz.de

JR: 0000-0003-0633-3386;; PT: 0000-0002-4672-2792

10.20982/tqmp.14.1.p017

Acting Editor $\square$ Denis Cousineau (Université d'Ottawa)

Reviewers - Julien T. Groulx (Université d'Ottawa)

- and one more.

\section{Introduction}

A valuable quality of the diffusion model is that it provides detailed information about the cognitive processes underlying performance on binary decision tasks regarding a variety of psychological phenomena (e. g., recognition memory, Spaniol, Madden, \& Voss, 2006; lexical decisions, Ratcliff, Thapar, Gomez, \& McKoon, 2004; perceptual discrimination, A. Voss, Rothermund, \& Voss, 2004; A. Voss, Rothermund, \& Brandstädter, 2008; priming effects, A. Voss, Rothermund, Gast, \& Wentura, 2013; faking, Röhner \& Ewers, 2016b; see also A. Voss, Nagler, \& Lerche, 2013). As such, it allows researchers to explain the observed data (i.e., reaction times and errors) in terms of psychologically meaningful processes (e. g., the ease of decision making, the response caution, and even nondecision-related processes). Along these lines, recent research by Klauer, Voss, Schmitz, and Teige-Mocigemba (2007) as well as by Röhner and Ewers (2016b) has shown that diffusion model analyses can be used to analyze and interpret the results of a very popular and frequently applied binary decision task (Bosson, Swann, \& Pennebaker, 2000; Rudolph, Schröder-Abé, Schütz, Gregg, \& Sedikides, 2008) called the Implicit Association Test (IAT; Greenwald, McGhee, \& Schwartz, 1998) in a much more fine-grained manner. 


\section{What is the IAT?}

The IAT was designed as a computerized categorization task to assess automatic implicit associations between two target concepts and one attribute dimension by using participants' observable data (i.e., reaction times and errors). Considered to assess automatic, uncontrollable, and thus, pure associations of participants even in socially sensitive areas (e. g., pedophilia, racism, stereotypes, or sexism; see e. g., Agerström \& Rooth, 2011; Banse, Schmidt, \& Clarbour, 2010; Banse, Seise, \& Zerbes, 2001; Carlsson \& Björklund, 2010; Gray, Brown, MacCulloch, Smith, \& Snowden, 2005; Greenwald et al., 1998; Greenwald \& Banaji, 1995; Latu et al., 2011), the IAT has of course attracted an enormous amount of research interest in recent years.

When taking the IAT, participants encounter stimuli that appear consecutively in the middle of the computer screen. They are asked to sort these stimuli into four different categories: (a) two contrasting target concept categories comprising the target dimension and (b) two contrasting attribute categories comprising the attribute dimension. The target dimension of an example IAT for the construct extraversion (Back, Schmukle, \& Egloff, 2009) includes self-relevant versus non-self-relevant words (e. g., me vs. others), and the attribute dimension includes extraversion-related versus introversion-related words (e. g., talkative vs. shy). As participants take the IAT, their reaction times and errors (i.e., wrongly categorized stimuli) are recorded by the computer.

The IAT consists of a total of seven blocks. Blocks 1, 2 , and 5 are the so-called single or practice blocks, which introduce the target or attribute discrimination. In these blocks, the categories of either the target concepts or the attribute concepts are presented in the upper left and right corners of the display screen. Participants are instructed to respond to exemplars of each category by pressing a key on the same side as the label. Blocks 3, 4, 6, and 7 are called the combined blocks. For these, the attribute discrimination is paired with the target discrimination (i.e., participants must assign words from all four categories in these blocks). Thus, on the extraversion IAT, in Blocks 3 and 4 (the compatible phase), participants must respond to selfrelevant and extraversion-related words with one key and to non-self-relevant and introversion-related words with the other key. In Blocks 6 and 7 (the incompatible phase), participants must respond to introversion-related and selfrelevant words with one key and to extraversion-related and non-self-relevant words with the other key.

The rationale behind the IAT is that the sorting task should be easier and thus completed more quickly when the two concepts that share one response key are strongly associated. If two concepts are only weakly associated, sorting them into one category should be more difficult and should be conducted more slowly. The presentation of the combined phases can be counterbalanced in the IAT (i.e., either the compatible phase is followed by the incompatible phase or vice versa). We present only the most common order here so that we can avoid unnecessarily complicating our description of the IAT.

\section{The traditional IAT effect and its possible contamination}

The traditional IAT effect (i.e., the so-called $D$ measure; see Greenwald, Nosek, \& Banaji, 2003a, 2003b) is computed as the difference in reaction times between the incompatible phase and the compatible phase divided by their overall standard deviation. It is used as an indicator of the strength of the association between the presented concepts (e. g., here, self and extraversion).

Although the IAT's validity for measuring automatic associations has been documented in a number of studies (e. g., Banse et al., 2001; Bar-Anan \& Nosek, 2014; Gawronski, 2002; Greenwald et al., 1998; Hofmann, Gawronski, Gschwendner, Le, \& Schmitt, 2005), its result might nevertheless be contaminated by factors other than the automatic associations that the IAT is supposed to assess. Research has shown that the traditional IAT effect (i.e., the $D$ measure; Greenwald et al., 2003a, 2003b) contains not only variance related to the construct but also methodspecific variance (e. g., Back, Schmukle, \& Egloff, 2005; McFarland \& Crouch, 2002; Mierke \& Klauer, 2003) and fakingrelated variance (e. g., De Houwer, Beckers, \& Moors, 2007; Fiedler \& Bluemke, 2005; McDaniel, Beier, Perkins, Goggin, \& Frankel, 2009; Röhner, Schröder-Abé, \& Schütz, 2011, 2013; Steffens, 2004). Here, the diffusion model comes into play.

\section{What is the Diffusion Model?}

The diffusion model (e. g., Ratcliff, 1978, 2014; Ratcliff, Gomez, \& McKoon, 2004; Ratcliff \& Rouder, 1998, 2000) represents a stochastic model for binary decision tasks. It is built on the assumption that people continuously accumulate information (i.e., response evidence) from the stimuli presented in a task in order to make decisions. After collecting sufficient information, they make their decision.

In Figure 1, a sample decision path for a fictional participant (we will call him Patrick) is represented in order to explain the process underlying the diffusion model. It distinguishes between two periods: the period of the actual decision process (represented by parameters $v$ and $a$ ) and the nondecision period (represented by parameter $t_{0}$ or $T_{e r}$ respectively; to simplify matters, we will use only the form $t_{0}$ hereafter). During the actual decision process, Patrick makes his decision on the basis of the information presented by the stimulus. The nondecision pe- 
Figure 1 - The diffusion process underlying the diffusion model (cf. Schmitz \& Voss, 2012). The diffusion model distinguishes between the nondecision parameter (parameter $t_{0}$ or $T_{e r}$, respectively) and parameters from the actual decision process (parameters $v$ and $a$ ). The X-axis represents time and is read from left to right. The Y-axis represents the responserelated decision with two response criteria which are placed at 0 (for incorrect responses) and $a$ (for correct responses). The counter begins fluctuating as a function of information that accumulates with time at the postulated point $z$. The accumulation of information includes systematic as well as random influences. As soon as one of the two response criteria is crossed, the decision process is terminated, and the corresponding response is initiated. In the sample path for one trial shown in the figure, the participant accumulates enough information to privide the correct response. Parameter $v$ is the mean amount of accumulated information for one participant across a certain number of trials and for a given type of stimuli. Distributions of correct and incorrect responses are displayed outside their respective response critera. This figure is reused from Röhner and Ewers (2016b). Copyright 2016 by the Psychonomic Society. All right reserved. Used with permission.

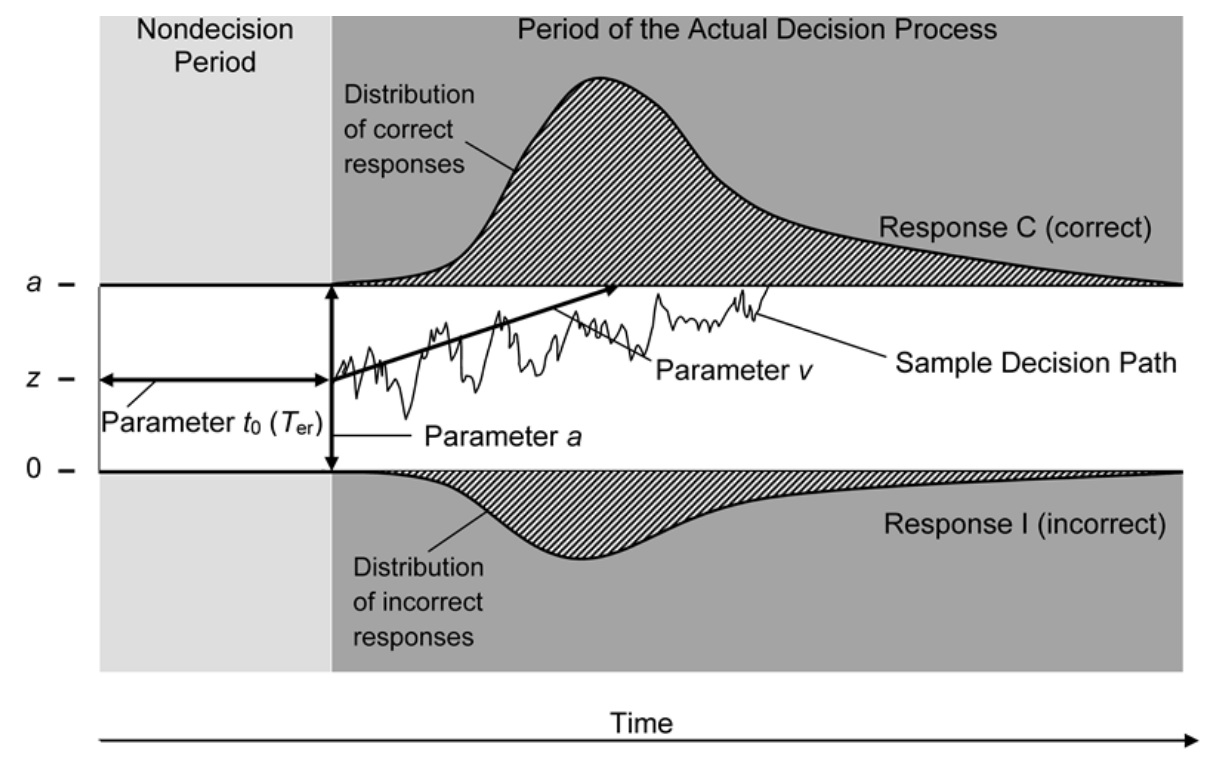

riod includes processes that are applied before and after the actual decision (e. g., Patrick's perceptual encoding of the stimulus and his motor execution of a key press to indicate his decision). The $\mathrm{X}$-axis represents time. The Y-axis represents the response-related decision axis.

Patrick's decision process begins at a certain starting point (i.e., point $z$ ). Beginning at this point, Patrick accumulates systematic and random information over time. Thus, his sample decision path runs like an internal counter that changes over time. The counter thereby runs in a corridor between two response criteria, which are located on the response-related decision axis. The two response criteria are placed at 0 and $a$. Each criterion represents one of the two response alternatives. In our example, the upper criterion (i.e., $a$ ) corresponds to the correct response (i.e., Response $\mathrm{C}$ ), whereas the lower criterion corresponds to the incorrect response (i.e., Response I). The counter is driven in opposite directions by informa- tion supporting the two different decisional outcomes (i.e., the response alternatives).

As soon as one of the two response criteria (i.e., the upper criterion $a$ or the lower criterion 0 ) is crossed, the decision process is terminated, and the response linked to the respective response criterion is initiated. For Patrick's sample decision path, the upper criterion is crossed, and thus, the correct response is initiated (see Klauer et al., 2007; Ratcliff, Thapar, Gomez, \& McKoon, 2004; Schmitz \& Voss, 2012). Decision tasks typically include more than one trial. After the first decision has been made, a second and even some additional decisions will usually follow. Patrick will, for example, provide the response related to the first decision and will then begin again by encoding the next stimulus by accumulating information until one of the criteria is satisfied. He will then provide the next response and so on. Parameter $v$ represents the mean amount of information that Patrick accumulates across a certain num- 
ber of decision processes. The distributions of correct and incorrect responses on several hypothetical trials are displayed outside their respective response criteria in Figure 1.

\section{The basic diffusion model versus the full diffusion model.}

The parameters that were mentioned above (i.e., parameters $v, a, t_{0}$, and $z$ ) are defined in the so-called basic diffusion model. The basic diffusion model can be extended by adding parameters that fit special research applications and allow for variability in trial-by-trial performances within a participant and an experiment. In this so-called full diffusion model, there are three additional parameters: (i) the intertrial variability in the (relative) starting point (i.e., parameter $s_{z r}$ ) that describes Patrick's trial-by-trial variation in the starting point relative to the threshold separation (see Ratcliff \& Rouder, 1998), (ii) the intertrial variability of the drift (i.e., parameter $s_{v}$ ) that describes Patrick's trial-by-trial variation in accumulation rate (see Ratcliff, 1978), and (iii) the intertrial variability of nondecisional components (i.e., parameter $s_{t_{0}}$ ) that describes Patrick's trial-by-trial variation in nondecision time (see Ratcliff \& Tuerlinckx, 2002). Both models also include a scaling parameter (i.e., parameter $s$ ) that quantifies a stochastic, nonsystematic component of the information accumulation process in each trial.

Whether the basic or the full diffusion model fits the data appropriately has to be carefully considered and decided by the researcher. For most research applications, the basic diffusion model might be sufficient (e. g., Van Ravenzwaaij, Donkin, \& Vandekerckhove, 2016) because it contains the most informative parameters $v, a$, and $t_{0}$ of the diffusion process (see Klauer et al., 2007; Ratcliff \& Rouder, 1998; Schmitz \& Voss, 2012; A. Voss et al., 2004), whose validities have furthermore already been successfully demonstrated in various experiments (e. g., Ratcliff, Thapar, Gomez, \& McKoon, 2004; Ratcliff, Thapar, \& McKoon, 2001; Thapar, Ratcliff, \& McKoon, 2003; A. Voss et al., 2004). By contrast, the additional intertrial variability parameters of the full diffusion model (i.e., parameters $s_{z r}, s_{v}$, and $s_{t_{0}}$ ) are usually not very reliable (Schmitz \& Voss, 2012) and require many trials in the order of about $N=1,000$ (A. Voss, Voss, \& Lerche, 2015) to be estimated reliably. For these reasons, we will describe only the three parameters $v, a$, and $t_{0}$ of the basic diffusion model in a more detailed manner, by referring to our fictional participant Patrick.

The most informative parameters of the basic diffusion model.

The construct parameter $\boldsymbol{v}$. Parameter $v$ refers to Patrick's performance in the actual decision process. It quantifies the efficacy with which Patrick accumulated response-related information. In other words, parameter $v$ describes how easy it was for Patrick to make a decision. The higher Patrick's value on parameter $v$, the faster Patrick reacted while simultaneously committing only a few errors, or stated more simply, the easier it was for Patrick to make his decision.

The response caution parameter $a$. Parameter $a$ refers to the amount of evidence Patrick accumulated before he made a decision in the actual decision process. It quantifies a nonability-related personality characteristic that contributes to speed-accuracy settings (Schmitz \& Voss, 2012): the so-called response caution. People differ in whether they prefer a conservative response mode (i.e., respond more slowly but with high accuracy) or a more liberal response mode (i.e., respond more quickly by accepting the risk of increased errors). The higher Patrick's value on parameter $a$, the more information he sampled before he made his decision, or stated more simply, the more conservative his response mode was.

The nondecision parameter $\boldsymbol{t}_{\mathbf{0}}$. Parameter $t_{0}$ refers to nondecision components in Patrick's reaction time (cf. Klauer et al., 2007; Schmitz \& Voss, 2012). This includes, for example, the perceptual encoding of the stimuli, task preparation, task switching, and the execution of motor responses (e. g., Klauer et al., 2007; Schmitz \& Voss, 2012). The last item in particular has found a great deal of empirical support (e. g., A. Voss et al., 2004; Ratcliff, Thapar, \& McKoon, 2006). The higher Patrick's value on parameter $t_{0}$, the more time he took to press a response key (e. g., due to his ability to execute this motor response).

\section{Can Diffusion Model analyses help in the interpreta- tion of IAT results?}

The traditional IAT effect (i.e., the $D$ measure; Greenwald et al., 2003a, 2003b) can be contaminated by method-specific variance (e. g., Back et al., 2005; McFarland \& Crouch, 2002; Mierke \& Klauer, 2003) and faking-related variance (e. g., De Houwer et al., 2007; Fiedler \& Bluemke, 2005; McDaniel et al., 2009). Klauer et al. (2007) therefore investigated whether diffusion model analyses can help to separate construct-related variance from method-related variance. They demonstrated that the parameters $v, a$, and $t_{0}$ of the diffusion model allow the researcher to compute three dissociable IAT effects (i.e., $I A T_{v}, I A T_{\mathrm{a}}$, and $\left.I A T_{t_{0}}\right)$ by subtracting the parameters that were estimated in the compatible phase from the parameters that were estimated in the incompatible phase in each case (i.e., $I A T_{v}$ = parameter $v$ incompatible phase - parameter $v$ compatible phase, $I A T_{\mathrm{a}}=$ parameter $a$ incompatible phase parameter $a$ compatible phase, and $I A T_{t_{0}}=$ parameter $t_{0}$ incompatible phase - parameter $t_{0}$ compatible phase).

The Quantitative Methods for Psychology 
Whereas $I A T_{v}$ was shown to be significantly related to the construct-related variance of the IAT, $I A T_{\mathrm{a}}$ was shown to be related to method-specific variance (Klauer et al., 2007). In other words: The diffusion-model-based IAT effects allow the construct-related variance to be separated from the method-specific variance. Klauer et al. (2007) supposed that $I A T_{\mathrm{a}}$ and $I A T_{t_{0}}$ may provide indications of faking.

Hereupon, Röhner and Ewers (2016b) investigated whether diffusion model analyses can be used to separate construct-related variance from faking-related variance. They investigated the impact of faking on the diffusionmodel-based IAT effects (i.e., $I A T_{v}, I A T_{\mathrm{a}}$, and $I A T_{t_{0}}$ ) that were computed from the estimated parameters $v, a$, and $t_{0}$ with fast-dm (A. Voss \& Voss, 2007, 2008). Therefore, Röhner and Ewers (2016b) used a total of four faking conditions (i.e., faking low vs. high scores and faking in a naïve vs. informed manner). Under so-called naïve faking conditions, participants were asked to fake high or low scores without any hints about how to do so, whereas under so-called informed faking, they were asked to fake high or low scores after receiving hints about how to do so. If faking-related variance can be completely separated from construct-related variance, faking should have had an impact on $I A T_{\mathrm{a}}$ and $I A T_{t_{0}}$ but not on $I A T_{v}$.

The results of this study demonstrated that diffusionmodel-based IAT effects are quite helpful for providing a more fine-grained interpretation of IAT effects. Although $I A T_{v}$ was impacted by faking in most faking conditions, and thus, it is not yet possible to simply remove the fakingrelated variance of the IAT effect, the results were nevertheless promising. The impact of faking on $I A T_{v}$ was much less pronounced under the easier informed faking conditions compared with the more difficult naïve faking conditions (cf. Röhner et al., 2013). Under the informed faking of low scores (i.e., the comparably easiest and most likely faking condition; see Röhner \& Ewers, 2016b; cf. Röhner et al., 2013), $I A T_{v}$ was even unaffected. In addition, $I A T_{\mathrm{a}}$ was impacted under this condition and thus might be useful as an indicator of faking attempts here. $I A T_{t_{0}}$ was not at all affected by faking. In sum, the impact of faking on the diffusion-model-based IAT effects depended on the faking conditions. Whereas naïve faking was not indicated at all, informed attempts to fake low might be indicated by $I A T_{\mathrm{a}}$.

According to the results by Klauer et al. (2007) as well as Röhner and Ewers (2016b), analyzing the IAT with diffusion model analyses is advantageous for better understanding the processes behind the IAT effect. Why do researchers not use diffusion model analyses more often as an additional tool that can enable them to interpret IAT results in a more fine-grained manner?

\section{Possible obstacles to using Diffusion Model analyses}

Compared with the quick and easy way to compute the IAT effect with the $D$ measure, analyzing IAT results with diffusion model analyses is of course much more complex and time-consuming (e. g., Röhner \& Ewers, 2016a, 2016b). Due to the complexity of the diffusion model, only a small circle of experts has used diffusion model analyses to analyze their data to date (e. g., A. Voss, Nagler, \& Lerche, 2013; Wagenmakers, van der Maas, \& Grasman, 2007; Wagenmakers, van der Maas, Dolan, \& Grasman, 2008). From an applied point of view, the increased complexity and the corresponding additional investments of effort and time might be the main reasons for why researchers from outside the "diffusion model universe" tend to abstain from using diffusion model analyses. In particular, there might be researchers who would like to use diffusion model analyses to investigate their IAT data in more detail, but if they are not yet familiar with diffusion model analyses, they might be deterred from doing so.

EZ: An easy and powerful way to apply Diffusion Model analyses

It is important to note that there are different tools that can be used to analyze data with diffusion modeling (e. g., DMAT, Vandekerckhove \& Tuerlinckx, 2007, 2008; EZ, Wagenmakers et al., 2007; EZ2, Grasman, Wagenmakers, \& van der Maas, 2009; robust-EZ, Wagenmakers et al., 2008; fast-dm, A. Voss \& Voss, 2007, 2008; HDDM, Wiecki, Sofer, \& Frank, 2013; HDM, Vandekerckhove, Tuerlinckx, \& Lee, 2011). They differ a lot with regard to what they require from the user. Among these tools, EZ can be considered an ideal tool to start with-especially for nonexperts in diffusion modeling (e. g., researchers who would like to easily and quickly analyze their IAT data in a more detailed manner with the help of diffusion modeling but have never done so before).

From a practical standpoint, EZ is easier to handle than the other tools for several reasons. First, most tools (DMAT, fast-dm, HDDM, and HDM) use the entire reaction time distribution for both correct and incorrect responses as input. Thus, the researcher has to invest time into putting those distributions into the requested format (see, e. g., Röhner \& Ewers, 2016a). EZ does not require that kind of input. Instead, it needs only three values that the researcher can compute quite quickly (i.e., the mean of the reaction times, the variance of the reaction times, and the percentage of correct responses) as input. Second, EZ estimates only the most informative parameters (i.e., parameter $v$, parameter $a$, and parameter $t_{0}$; see also Klauer et al., 2007; Ratcliff \& Rouder, 1998; Schmitz \& Voss, 2012; A. Voss et al., 2004). In other words, it forgoes some parameters from 
Ratcliff's diffusion model (i.e., parameter $z$, which is fixed to $a / 2$ in EZ, parameter $s_{z r}$, parameter $s_{v}$, and parameter $s_{t_{0}}$; see, e. g., Wagenmakers et al., 2007). Thus, the number of parameters to be interpreted is reduced to a minimum and seems much more manageable, especially for novices in diffusion modeling. Third, no optimization or fitting routine is necessary, which provides two strong benefits for practical implementations. On the one hand, it means that the researcher does not need to be familiar with complex fitting routines (e. g., Van Ravenzwaaij et al., 2016). On the other hand, the researcher can obtain the parameters immediately (Wagenmakers et al., 2007). Altogether, EZ seems to be an ideal tool for first-time users to conduct diffusion model analyses.

EZ has been criticized for being too easy because it operates at a more macroscopic level and might not capture all aspects of reality that one might consider important (cf. Ratcliff, 2008). Research by Van Ravenzwaaij and Oberauer (2009), however, demonstrated that EZ, when compared with other tools (i.e., DMAT and fast-dm), does a considerably better job at recovering the most informative parameters $v, a$, and $t_{0}$ when confronted with a small number of trials and thus is sometimes more capable of capturing experimental effects on the parameters. In addition, Van Ravenzwaaij and Oberauer (2009) indicated that EZ, in comparison with fast-dm, is associated with more statistical power to detect significant effects. Fast-dm was sometimes found to yield smaller differences between the experimental conditions than actually existed (Van Ravenzwaaij \& Oberauer, 2009).

Summing up, recent research has also shown that not only is the EZ diffusion model easy to use (e. g., Grasman et al., 2009), but it also provides a very powerful test of simple experimental effects (e. g., Van Ravenzwaaij et al., 2016). Thus, although EZ might not be applicable to the broad range of data patterns for which other tools including the full diffusion model will be more appropriate, EZ will by virtue of its simplicity sometimes be even better at detecting effects (see Van Ravenzwaaij et al., 2016).

\section{The present study}

Given that (a) the IAT represents a very popular measure (Bosson et al., 2000; Rudolph et al., 2008), (b) its fakeability has already been well-documented (e. g., Fiedler \& Bluemke, 2005; Röhner et al., 2011, 2013; Steffens, 2004), (c) diffusion model analyses are advantageous for interpreting IAT results (Klauer et al., 2007; Röhner \& Ewers, 2016b), and (d) most likely the main obstacle that prevents researchers from using diffusion model analyses is the complexity of such analyses (e. g., A. Voss, Rothermund, Gast, \& Wentura, 2013; Wagenmakers et al., 2007; Wagenmakers et al., 2008), an important question is whether EZ might be a tool that combines the advantage of being an easy diffusion model analysis to apply with the advantage of offering a better interpretation of IAT results. Previous research has focused on fast-dm as one way to compute and analyze IAT effects with diffusion model analyses (e. g., Röhner \& Ewers, 2016b). Thus far, EZ has not been used, although it can be considered to be associated with, on the one hand, especially quick and easy diffusion modeling even for nonexperts, and on the other hand, high statistical power in detecting the impact of experimental manipulations on the model parameters (Van Ravenzwaaij \& Oberauer, 2009). Based on its characteristic of combining easy and quick diffusion model analyses as well as the powerful detection of the impact on the model parameters, it is relevant to investigate whether EZ can be used to analyze and interpret IAT results in a more fine-grained manner. For these reasons, we used EZ to reanalyze a data set of faked and nonfaked IAT effects. Our study's hypotheses are summarized as follows.

1. Because $I A T_{v}$ was impacted by naïve faking when it was computed on parameter $v$ in the compatible and incompatible IAT phases when estimated with fast-dm (see Röhner \& Ewers, 2016b), we expected that $I A T_{v}$ would also be impacted by naïve faking when computed on parameter $v$ in the compatible and incompatible IAT phases when estimated with $E Z$. We expected $I A T_{v}$ to be less impacted by faking attempts under informed faking conditions when computed on parameter $v$ in the compatible and incompatible IAT phases when estimated with $E Z$ because of the results that have been found for $I A T_{v}$ on parameter $v$ in the compatible and incompatible IAT phases when estimated with fast-dm.

2. Because $I A T_{\mathrm{a}}$ was impacted by informed faking of low scores when it was computed on parameter $a$ in the compatible and incompatible IAT phases when estimated with fast-dm (see Röhner \& Ewers, 2016b), we expected that $I A T_{\mathrm{a}}$ would also be impacted by informed faking of low scores when computed on parameter $a$ in the compatible and incompatible IAT phases when estimated with $E Z$. We did not expect an impact of naïve faking of low and high scores or an impact of informed faking of high scores on $I A T_{\mathrm{a}}$ when computed on parameter $a$ in the compatible and incompatible IAT phases when estimated with $E Z$ because $I A T_{\text {a }}$ was also not impacted under these conditions when it was computed on parameter $a$ in the compatible and incompatible IAT phases when estimated with fast-dm.

3. Because $I A T_{t_{0}}$ was not impacted by any faking attempts (i.e., faking low or high scores under naïve or informed faking) when it was computed on parameter $t_{0}$ in the compatible and incompatible IAT phases when 
estimated with fast-dm (see Röhner \& Ewers, 2016b), we expected that $I A T_{t_{0}}$ would also not be influenced by faking when computed on parameter $t_{0}$ in the compatible and incompatible IAT phases when estimated with $E Z$.

\section{Method}

\section{Data for reanalysis}

In order to be able to compare the results of EZ with the results of fast-dm, we reanalyzed the published data set that was already used to examine the process components in the IAT with fast-dm by Röhner and Ewers (2016b) and that was originally collected to investigate the behavior of fakers on the IAT (Röhner et al., 2013). In the data set from Röhner et al. (2013), a total of 84 volunteers (64 female; 74 students) from Chemnitz University of Technology participated in the study in exchange for personal feedback and partial course credit (see Röhner et al., 2013). Their mean age was 22.37 years $(S D=4.45)$.

Participants were randomly assigned to one of three conditions: (a) a control group, (b) a faking condition $\mathrm{LH}$ (faking low scores first and high scores second), or (c) a faking condition HL (faking high scores first and low scores second). All participants completed an extraversion IAT (Back et al., 2009) a total of three times. First, they completed the IAT once without faking instructions (i.e., baseline assessment). Participants in the control group then completed the IAT two more times without further instructions. Participants in the faking conditions were asked to fake the IAT first with no information about the IAT's rationale or faking strategies (naïve faking). Afterwards, they were told how to fake the IAT and were asked to fake the IAT again (informed faking). Participants in the LH faking condition faked low scores under the naïve faking condition and high scores under the informed faking condition. Participants in the HL faking condition faked high scores under the naïve faking condition and low scores under the informed faking condition.

\section{Analytical approach}

We used the Excel sheet from the EZ software, which can be downloaded (see http://www.ejwagenmakers.com/papers. html). Note that a JavaScript, R Code, and a MATLAB implementation are also available on this site. Also note that it is possible to download the EZ2 software and the robust-EZ software. We used the $E Z$ software for our analyses. In the Excel sheet, the specific value of parameter $s$ can be chosen. Note that parameter $s$ represents a scaling parameter. In other words, changes in the $s$ parameter lead to changes in other parameters (e. g., if $s$ doubles, other parameters double accordingly). In practice, parameter $s$ is usually set to 0.1 . We followed this convention in our analyses.

Pretreatment of the data set. In order to keep the input for the diffusion model analyses identical to that of Röhner and Ewers (2016a, 2016b), we followed the recommendation by A. Voss, Rothermund, Gast, and Wentura (2013) as well as A. Voss and Voss (2008) to remove outliers from the individual response-time distribution if participants had reaction times below $200 \mathrm{~ms}$ or above $5,000 \mathrm{~ms}$. We excluded 156 trials (i.e., $0.3 \%$ of the trials). Because we set parameter $s$ to the conventional value of 0.1 , we accordingly transformed our reaction time data from milliseconds to seconds before further analyses.

Computation of the input variables. We computed the mean reaction time, the variance of the reaction times, and the percentage of correct responses separately for each participant ( $N=84$ ) and each combined IAT phase type (i.e., compatible vs. incompatible phase) within every measurement occasion (i.e., baseline, retest/naïve faking, and retest/informed faking). Two participants had to be excluded from further analyses due to their percentage of correct responses of exactly 0.5 .

Parameter estimation. We used EZ to estimate the independent EZ diffusion models for each participant and each combined IAT phase type within every measurement occasion. Altogether, we computed $492 \mathrm{EZ}$ diffusion model analyses (i.e., 82 participants $\times 2$ phase types $\times 3$ measurement occasions). Each diffusion model analysis was based on about 96 trials (i.e., exactly 96 trials in the absence of outlier trials and an average of 92 trials when outliers were excluded).

Computation of the compatibility effects. On the basis of the parameters estimated in the EZ diffusion model analyses, we computed the compatibility effects $I A T_{v}, I A T_{\mathrm{a}}$, and $I A T_{t_{0}}$. We did so by subtracting the parameters that were estimated in the compatible phase from the parameters that were estimated in the incompatible phase (i.e., $I A T_{v}=$ parameter $v$ incompatible phase - parameter $v$ compatible phase, $I A T_{\mathrm{a}}=$ parameter $a$ incompatible phase - parameter $a$ compatible phase, and $I A T_{t_{0}}=$ parameter $t_{0}$ incompatible phase - parameter $t_{0}$ compatible phase; see Klauer et al., 2007). Because of this computation of the compatibility effects, negative values on $I A T_{v}$ indicate a strong association between the self and extraversion and positive values on $I A T_{v}$ indicate a weak association between the self and extraversion.

Analyzing the compatibility effects and the parameters. After computing the input variables for the EZ diffusion model analyses (i.e., the mean reaction time, the variance of the reaction times, and the percentage of correct responses), estimating the parameters, and computing the compatibility effects, we used repeated-measures ANOVAs to test our hypotheses. For all ANOVAs, we set $\alpha$

The Quantitative Methods for Psychology 
to .05. First, we computed 3 (measurement occasion) $\times$ 3 (experimental group) ANOVAs with repeated measures on the respective compatibility effects (i.e., $I A T_{v}, I A T_{\mathrm{a}}$, and $I A T_{t_{0}}$; one for each measurement occasion) to investigate whether faking would have an impact on those IAT effects. Second, to take a closer look at the results, we additionally computed 3 (measurement occasion) $\times 3$ (experimental group) ANOVAs with repeated measures on the parameters (i.e., parameters $v, a$, and $t_{0}$; one for each measurement occasion) separately for the compatible and incompatible phases in each case. For all ANOVAs, we used Bonferroni-adjusted post hoc analyses.

\section{Power analyses}

To estimate the power of our analyses, we used G*Power 3.1.9.2 (Faul, Erdfelder, Lang, \& Buchner, 2007). Post hoc power analyses with $N=82$ and estimated correlations between measurements of $r=-.18$ and $r=.24$ revealed that a repeated-measures ANOVA had more than $83 \%(.825$ and .961) power to detect a moderate effect size. The correlations of $r=-.18$ and $r=.24$ represent the minimum and maximum values of correlations within measurement occasions that we obtained for the respective compatibility effects (see Table 1).

\section{Results}

To reduce complexity, we hereafter refer to parameters and IAT effects that were obtained with EZ as EZ-based parameters and EZ-based IAT effects and to those that were obtained with fast-dm as fast-dm-based parameters and fast-dm-based IAT effects. To determine whether faking affected $I A T_{v}, I A T_{\mathrm{a}}$, and $I A T_{t_{0}}$ when computed with the EZ diffusion model parameters, we computed 3 (measurement occasion) $\times 3$ (experimental group) repeatedmeasures ANOVAs separately for $I A T_{v}, I A T_{\mathrm{a}}$, and $I A T_{t_{0}}$. To take a closer look at the results, we additionally computed 3 (measurement occasion) $\times 3$ (experimental group) repeated-measures ANOVAs separately for parameter $v$, parameter $a$, and parameter $t_{0}$ and separately for the compatible and incompatible phases for each parameter (for a total of six ANOVAs). To facilitate the comparisons of the results between EZ and fast-dm, Table 2 shows the main effects and interaction effects with the corresponding effect sizes for the EZ-based as well as for the comparisons with the fast-dm-based IAT effects and parameters. Corresponding between- and within-group comparisons can be found in Tables 3, 4, and 5.

\section{Effects of faking on the EZ-based I $A T_{v}$}

As expected, the results were very similar to the results that were obtained for the fast-dm-based $I A T_{v}$ (see Table 2). For the EZ-based $I A T_{v}$ the interaction effect,
$F(3.77,149.04)=26.31, p<.001, \omega^{2}=.54$, was large in size and significant, whereas the main effects of group, $F(2,79)=2.13, p=.126, \omega^{2}=.03$, and measurement occasion, $F(1.89,149.04)=1.28, p=.281, \omega^{2}=.01$, were nonsignificant. The expected interaction effect was comparably strong between the EZ-based and the fast-dmbased $I A T_{v}$ (i.e., large effect each time). Congruent with the results for the fast-dm-based $I A T_{v}$ (Röhner \& Ewers, 2016b), the naïve faking of low and high scores and the informed faking of high scores had an influence on the EZ-based $I A T_{v}$ (i.e., $I A T_{v}$ increased or decreased in accordance with the requested faking direction; low or high scores, respectively; see Table 3; Figure 2). Also in line with the results for the fast-dm-based $I A T_{v}$, the impact on the EZ-based $I A T_{v}$ was more pronounced under naïve than under informed faking. Unexpectedly, the informed faking of low scores also had an impact on the EZ-based $I A T_{v}$ (i.e., it significantly increased), which was not the case for the fast-dm-based $I A T_{v}$.

Similar to the results from previous analyses driven by the fast-dm-based parameters $v$ in the compatible phase, for the EZ-based parameters $v$ in the compatible phase, the main effects of measurement occasion, $F(1.98,156.61)=$ $35.47, p<.001, \omega^{2}=.45$, and group, $F(2,79)=$ $8.99, p<.001, \omega^{2}=.16$, as well as the interaction effect, $F(3.97,156.61)=59.45, p<.001, \omega^{2}=.74$, were significant (Table 2). Comparing the effect sizes on a descriptive level, they were somewhat larger for the EZ-based parameters (i.e., large) than for the fast-dm-based parameters (i.e., medium to large). Also similar to the results for the fast-dm-based parameter (Röhner \& Ewers, 2016b), for the EZ-based parameter $v$ in the incompatible phase, the main effects of measurement occasion, $F(1.92,151.69)=$ $22.61, p<.001, \omega^{2}=.34$, and the interaction effect, $F(3.84,151.69)=5.34, p=.001, \omega^{2}=.17$, were significant. The main effect of group, $F(2,79)=4.07, p=$ $.021, \omega^{2}=.07$, was significant for the EZ-based parameter $v$ in the incompatible phase, but has been nonsignificant for the fast-dm-based one. Again, the effect sizes for the EZ-based parameters were somewhat larger, ranging from medium to large, compared with the fast-dm-based parameters, which ranged from small to medium.

Taken together, EZ and fast-dm led, as expected, to very comparable results regarding the impact of faking on $I A T_{v}$ and parameters $v$ in the compatible and incompatible IAT phases, whereby the effect sizes were descriptively somewhat more pronounced when EZ was used.

\section{Effects of faking on the EZ-based I AT}

Regarding the EZ-based $I A T_{\mathrm{a}}$, the main effect of group, $F(2,79)=5.18, p=.008, \omega^{2}=.09$, and the interaction, $F(3.89,153.72)=8.33, p<.001, \omega^{2}=.26$, were medium 
Table 1 a Correlations Within Measurement Occasions for the EZ-Based IAT-Effects

\begin{tabular}{|c|c|c|c|c|}
\hline & \multicolumn{4}{|c|}{$I A T_{v}$} \\
\hline & MO & B & $\mathrm{R} / \mathrm{NF}$ & $\mathrm{R} / \mathrm{IF}$ \\
\hline \multirow{4}{*}{$I A T_{v}$} & B & - & $.24^{*}$ & .21 \\
\hline & $\mathrm{R} / \mathrm{NF}$ & $.24 *$ & - & -.18 \\
\hline & $\mathrm{R} / \mathrm{IF}$ & .21 & -.18 & - \\
\hline & \multicolumn{4}{|c|}{$I A T_{\mathrm{a}}$} \\
\hline \multirow{5}{*}{$I A T_{\mathrm{a}}$} & MO & B & $\mathrm{R} / \mathrm{NF}$ & $\mathrm{R} / \mathrm{IF}$ \\
\hline & B & - & $.22 *$ & .14 \\
\hline & $\mathrm{R} / \mathrm{NF}$ & $.22 *$ & - & -.12 \\
\hline & $\mathrm{R} / \mathrm{IF}$ & .14 & -.12 & - \\
\hline & \multicolumn{4}{|c|}{$I A T_{t_{0}}$} \\
\hline \multirow{4}{*}{$I A T_{t_{0}}$} & MO & B & $\mathrm{R} / \mathrm{NF}$ & $\mathrm{R} / \mathrm{IF}$ \\
\hline & B & - & .06 & .02 \\
\hline & $\mathrm{R} / \mathrm{NF}$ & .06 & - & .04 \\
\hline & $\mathrm{R} / \mathrm{IF}$ & .02 & .04 & - \\
\hline
\end{tabular}

Note. MO represents measurement occasion; B represents baseline; R/NF represents retest/naïve faking; R/IF represents retest/informed faking; $N=82 ; p<.05$, two-tailed.

to large in size and significant, whereas the main effect of measurement occasion, $F(1.95,153.72)=0.73, p=$ $.479, \omega^{2}=.00$, was nonsignificant. This was, as hypothesized, quite comparable to what was found for the fast-dmbased $I A T_{\mathrm{a}}$. The expected interaction effect was descriptively somewhat larger for the EZ-based $I A T_{\mathrm{a}}$ (i.e., large) than for the fast-dm-based $I A T_{\mathrm{a}}$ (i.e., medium). In line with the results for the fast-dm-based $I A T_{\mathrm{a}}$, the EZ-based $I A T_{\mathrm{a}}$ expectedly was not impacted by the naïve faking of low and high scores (see Table 4; Figure 3) and expectedly decreased significantly when informed participants were asked to fake low scores. Interestingly, the EZ-based $I A T_{\mathrm{a}}$, in contrast to the fast-dm-based $I A T_{\mathrm{a}}$, also increased significantly when informed participants were asked to fake high scores.

For the EZ-based parameter $a$ in the compatible phase, the main effect of measurement occasion, $F(1.87,147.44)=27.55, p<.001, \omega^{2}=.38$, and the interaction effect, $F(3.73,147.44)=3.52, p=.011, \omega^{2}=$ .10 , were medium to large in size and significant. The main effect of group, $F(2,79)=1.14, p=.324, \omega^{2}=.00$, was nonsignificant. For the EZ-based parameter $a$ in the incompatible phase, the main effect of measurement occasion, $F(1.91,151.19)=37.49, p<.001, \omega^{2}=.46$, and the interaction effect, $F(3.38,151.19)=3.86, p=.006, \omega^{2}=$ .11 , were medium to large in size and significant. The main effect of group, $F(2,79)=0.22, p=.806, \omega^{2}=.00$, remained nonsignificant. The results are mostly comparable to what was found with the fast-dm-based analyses. With respect to the effect sizes, the EZ-based parameters for the compatible and incompatible phases were mostly somewhat higher (i.e., medium to large) than they were for the respective fast-dm-based parameters (i.e., small to medium). The results of the between- and within-group comparisons for the EZ-based parameter $a$ document the influence of the informed faking of low and high scores on $I A T_{\mathrm{a}}$. The informed faking of low scores and high scores led to a mostly significant decrease in the EZ-based parameter $a$ in the compatible phase and in the incompatible phase (see Table 4).

Summing up, as expected, the results regarding $I A T_{\mathrm{a}}$ and parameter $a$ in the compatible and incompatible IAT phases were very comparable between fast-dm and EZ. As was the case for $I A T_{v}$, the impact on $I A T_{\mathrm{a}}$ was more pronounced when computed with the EZ-based parameters.

\section{Effects of faking on the EZ-based IAT $T_{t_{0}}$}

The differences between the EZ-based $I A T_{t_{0}}$ and the fast$\mathrm{dm}$-based $I A T_{t_{0}}$ were larger (Table 2). Unexpectedly and in contrast to the analyses for the fast-dm-based $I A T_{t_{0}}$, for the EZ-based $I A T_{t_{0}}$, the main effect of group, $F(2,79)=$ $9.66, p<.001, \omega^{2}=.17$, and the interaction effect, $F(3.93,155.39)=9.23, p<.001, \omega^{2}=.28$, were large in size and significant. The main effect of measurement occasion, $F(1.97,155.39)=0.93, p=.395, \omega^{2}=.00$, was nonsignificant. Accordingly, effect sizes for the significant effects were comparably higher for the EZ-based $I A T_{t_{0}}$ than for the fast-dm-based $I A T_{t_{0}}$ analyses (i.e., large vs. small to medium). The results for the between- and withingroup comparisons (see Table 5; Figure 4) might explain why there were differences. Congruent with the results obtained for the fast-dm-based parameters (Röhner \& Ew- 
Figure 2 - The means with corresponding error bars for the EZ-based $I A T_{v}$ and for the EZ-based parameters $v$ in the compatible and incompatible IAT phase, as well as for the fast-dm-based $I A T_{v}$ and for the fast-dm-based parameters $v$ in the compatible and incompatible IAT phase.

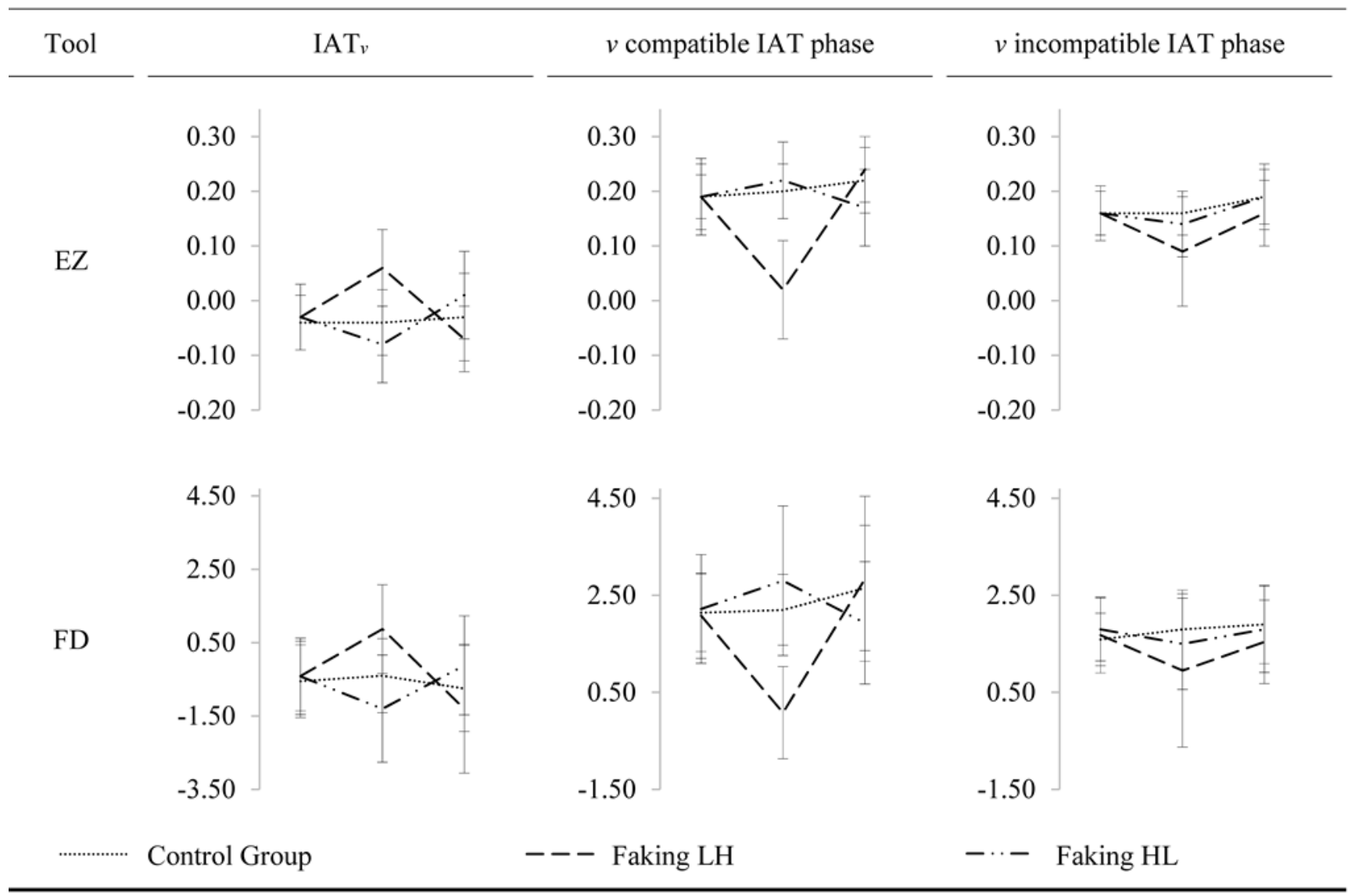

ers, 2016b), there were no significant differences between and within the groups on the EZ-based $I A T_{t_{0}}$ under the naïve faking of high and low scores. However, by contrast, there were significant differences between and within the groups on the EZ-based $I A T_{t_{0}}$ under the informed faking of high and low scores. The informed faking of high scores led to a significant increase in the EZ-based $I A T_{t_{0}}$ whereas the informed faking of low scores led to a significant decrease in it.

The results for the EZ-based parameter $t_{0}$ in the compatible and incompatible phases also differed from those obtained with the fast-dm-based parameter $t_{0}$ in the compatible and incompatible phases (Table 2). For the EZ-based parameter $t_{0}$ in the compatible phase, the main effect of measurement occasion, $F(1.92,151.25)=$ $9.83, p<.001, \omega^{2}=.17$, and the interaction effect, $F(3.83,151.25)=16.25, p<.001, \omega^{2}=.42$, were significant and large in size. The main effect of group,
$F(2,79)=2.56, p=.083, \omega^{2}=.04$, was nonsignificant. For the EZ-based parameter $t_{0}$ in the incompatible phase, the main effects of measurement occasion, $F(1.88,148.68)=12.26, p<.001, \omega^{2}=.21$, and group, $F(2,79)=4.41, p=.015, \omega^{2}=.08$, were significant and medium to large in size. The interaction, $F(3.76,148.68)=1.39, p=.242, \omega^{2}=.02$, remained nonsignificant. Consequently, the effect sizes were also comparably higher for the EZ-based parameter $t_{0}$ than for the fast-dm-based parameter $t_{0}$.

The impact of the informed faking of low and high scores on EZ-based $I A T_{t_{0}}$ was also visible in the betweenand within-group comparisons for the EZ-based parameter $t_{0}$ (Table 5). The informed faking of high scores led to a significant increase in the EZ-based parameter $t_{0}$ in the incompatible phase and to a nonsignificant but descriptive decrease in the EZ-based parameter $t_{0}$ in the compatible phase (see Table 5). The informed faking of low scores led 
Figure 3 - The means with corresponding error bars for the EZ-based $I A T_{a}$ and for the EZ-based parameters $a$ in the compatible and incompatible IAT phase, as well as for the fast-dm-based $I A T_{a}$ and for the fast-dm-based parameters $a$ in the compatible and incompatible IAT phase.

\begin{tabular}{|c|c|c|c|c|c|c|}
\hline Tool & & $\operatorname{IAT}_{a}$ & \multicolumn{2}{|c|}{$a$ compatible IAT phase } & \multicolumn{2}{|c|}{$a$ incompatible IAT phase } \\
\hline \multirow{6}{*}{$\mathrm{EZ}$} & 0.30 & \multirow{6}{*}{$\bar{E}=\ldots$} & 0.30 & \multirow{6}{*}{$=-2 x-m$} & 0.30 & \multirow{6}{*}{ 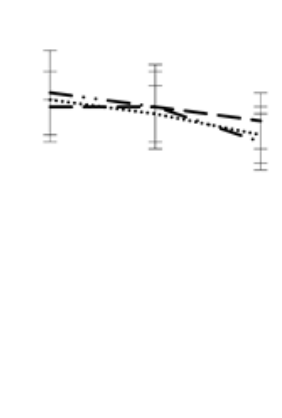 } \\
\hline & 0.20 & & 0.20 & & 0.20 & \\
\hline & 0.10 & & 0.10 & & 0.10 & \\
\hline & 0.00 & & 0.00 & & 0.00 & \\
\hline & -0.10 & & -0.10 & & -0.10 & \\
\hline & -0.20 & & -0.20 & & -0.20 & \\
\hline & 4.50 & & 4.50 & & 4.50 & \\
\hline & 2.50 & & 2.50 & I & 2.50 & \\
\hline FD & 0.50 & & 0.50 & & 0.50 & \\
\hline & -1.50 & & -1.50 & & -1.50 & \\
\hline & -3.50 & & -3.50 & & -3.50 & \\
\hline$\ldots \ldots \cdots$ & Control & & $---\mathrm{Fal}$ & ng LH & $-\cdots-$ & Faking HL \\
\hline
\end{tabular}

to a significant increase in the EZ-based parameter $t_{0}$ in the compatible phase and a significant increase in the EZbased parameter $t_{0}$ in the incompatible phase.

Thus, although the results were mostly similar between the EZ-based and the fast-dm-based $I A T_{t_{0}}$, they differed with respect to one condition (i.e., the informed faking condition). $I A T_{t_{0}}$ was impacted when it was computed with the EZ-based $t_{0}$ parameter but not when it was computed with the fast-dm-based $t_{0}$ parameter.

\section{Discussion}

Are diffusion model analyses when run with EZ helpful for analyzing and interpreting IAT results? We conducted this study to try to answer this question. The results showed that as hypothesized, diffusion model analyses, when computed with EZ, deliver results that are quite comparable to those obtained with fast-dm (Röhner \& Ewers, 2016b). EZ thereby seems to be somewhat more powerful in detecting the impact of faking on parameters $a$ and $t_{0}$ and the corresponding IAT effects. The results underpin the advantages of using diffusion-model-based IAT effects in order to obtain a more fine-grained picture of the components of the IAT result.

\section{Faking impacts the EZ-based $I A T_{v}$}

As expected, the EZ-based $I A T_{v}$ was impacted by faking under the naïve faking of low and high scores as well as under the informed faking of high scores. These results are identical to the results found by Röhner and Ewers (2016b) who used the fast-dm-based $I A T_{v}$ for their analyses. In contrast to Röhner and Ewers' (2016b) results and thus unexpectedly, $I A T_{v}$ was also somewhat impacted by the informed faking of low scores. In addition, the effects in general increased somewhat when the EZ parameters 
Figure 4 घ The means with corresponding error bars for the EZ-based $I A T_{t_{0}}$ and for the EZ-based parameters $t_{0}$ in the compatible and incompatible IAT phase, as well as for the fast-dm-based $I A T_{t_{0}}$ and for the fast-dm-based parameters $t_{0}$ in the compatible and incompatible IAT phase.

\begin{tabular}{|c|c|c|c|c|c|c|}
\hline Tool & & $\mathrm{IAT}_{t_{0}}$ & \multicolumn{2}{|c|}{$t_{0}$ compatible IAT phase } & \multicolumn{2}{|c|}{$t_{0}$ incompatible IAT phase } \\
\hline \multirow{5}{*}{ EZ } & 0.70 & \multirow{5}{*}{ F } & \multicolumn{2}{|l|}{0.70} & \multicolumn{2}{|l|}{0.70} \\
\hline & 0.40 & & 0.40 & & 0.40 & \multirow{2}{*}{ 手- } \\
\hline & 0.10 & & 0.10 & & 0.10 & \\
\hline & -0.20 & & -0.20 & & -0.20 & \\
\hline & -0.50 & & -0.50 & & -0.50 & \\
\hline \multirow{5}{*}{ FD } & 0.70 & \multirow{5}{*}{$=-1$} & \multicolumn{2}{|l|}{0.70} & \multicolumn{2}{|l|}{0.70} \\
\hline & 0.40 & & 0.40 & $z_{-1}$ & 0.40 & \\
\hline & 0.10 & & 0.10 & & 0.10 & \\
\hline & -0.20 & & -0.20 & & -0.20 & \\
\hline & -0.50 & & -0.50 & & -0.50 & \\
\hline 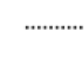 & \multicolumn{2}{|c|}{ Control Group } & \multicolumn{2}{|c|}{--- Faking LH } & $-\cdots-$ & Faking HL \\
\hline
\end{tabular}

instead of the fast-dm parameters were used. Both observations might be explained by research that demonstrated that EZ provides a very powerful test of simple experimental effects (Van Ravenzwaaij et al., 2016) and that fast-dm, when compared with EZ, sometimes yields smaller differences between experimental conditions than actually exist (Van Ravenzwaaij \& Oberauer, 2009).

The results replicate previous findings and emphasize that it is not yet possible to simply separate construct- and faking-specific variance from each other by using diffusion model analyses. Replicating the finding that parameter $v$ and thus $I A T_{v}$ are affected by faking also underpins the idea that faking might to some extent result from temporary changes in people's accessible mental associations (i.e., People can try to take on the role of an extraverted person by telling themselves: "While taking this test, I will be an extraverted person" or the role of an introverted person by telling themselves: "While taking this test, I will be an introverted person”; see Röhner \& Ewers, 2016b). Such temporary mental associations may be helpful for faking by causing a temporary change in mental associations. This change in mental associations might be one component of successful faking on the IAT because it influences the ease of decision-making on the IAT. Because people's faking intentions contribute to the ease of decisionmaking (i.e., parameter $v$ ), under faking, $I A T_{v}$ contains construct-related variance and faking-related variance.

Informed, but not naïve faking, impacts the EZ-based I $A T_{\mathrm{a}}$

Congruent with the results found by Röhner and Ewers (2016b) who used the fast-dm-based $I A T_{\mathrm{a}}$ for their analyses, the EZ-based $I A T_{\mathrm{a}}$ was not impacted by faking attempts under the naïve faking of low and high scores. Also, as expected, it was impacted by faking attempts under the informed faking of low scores. Incongruent with the re- 
sults that occurred when the fast-dm-based $I A T_{\mathrm{a}}$ was used (see Röhner \& Ewers, 2016b), the EZ-based $I A T_{\mathrm{a}}$ was also impacted by the informed faking of high scores. Although somewhat surprising, this difference might again be explained by the higher statistical power that is associated with EZ (see Van Ravenzwaaij et al., 2016).

Actually, the impact of faking on $I A T_{\mathrm{a}}$ is in line with the theoretical assumption that faking strategies (i.e., the acceleration or slowing down of reaction times in certain IAT phases; see Röhner et al., 2013) might be caused by the adaption of speed-accuracy settings (see Fiedler \& Bluemke, 2005). To fake low, fakers might slow down on the compatible phase by applying a higher response caution on that phase than on the incompatible one (see Fiedler \& Bluemke, 2005; Klauer et al., 2007). This results in a higher parameter $a$ in the compatible phase, a lower parameter $a$ in the incompatible phase, and thus, a decreased $I A T_{\mathrm{a}}$. To fake high scores, fakers might do exactly the opposite, which leads to an increased $I A T_{\mathrm{a}}$. This is exactly what the results of the current study look like -at least for informed faking. These changes in $I A T_{\mathrm{a}}$ emphasize that when people know how to fake the IAT, not only $I A T_{v}$ but also $I A T_{\mathrm{a}}$ is impacted, thus giving researchers some indication about faking. Given that the $D$ measure does not give any hints about faking, the current results suggest a clear benefit of diffusion-model-based IAT effects. Naïve faking did not impact $I A T_{\mathrm{a}}$, a result that underpins the idea that naïve and informed faking are implemented by participants in different ways (see Röhner \& Ewers, 2016b; see also Röhner et al., 2013).

\section{Informed, but not naïve faking, impacts the EZ-based $\boldsymbol{I} \boldsymbol{A} \boldsymbol{T}_{t_{0}}$}

Identical to Röhner and Ewers' (2016b) results from the fast-dm-based $I A T_{t_{0}}$, the EZ-based $I A T_{t_{0}}$ was not impacted by faking under the naïve faking of low and high scores. It is interesting that the EZ-based $I A T_{t_{0}}$, in contrast to the fast-dm-based $I A T_{t_{0}}$, was impacted by the informed faking of low and high scores. The impact of informed faking on $I A T_{t_{0}}$ might have been somewhat underestimated with fast-dm (see Van Ravenzwaaij \& Oberauer, 2009). Besides this statistical explanation, the finding is also in line with theoretical assumptions that faking strategies might be caused by motor-response adaptions that occur outside of the actual decision process (see Klauer et al., 2007). To fake low, fakers might slow down on the compatible phase by delaying their response execution in that phase more than in the incongruent one, resulting in a higher $t_{0}$ parameter in the compatible phase than in the incompatible phase and thus a decreased $I A T_{t_{0}}$ (see Röhner \& Ewers, 2016b). To fake high scores, fakers might do exactly the opposite, thus resulting in an increased $I A T_{t_{0}}$ (see Röhner \&
Ewers, 2016b). The results of the current study support this idea, at least for informed faking and supports previous assumptions that when people know how to fake the IAT, not only $I A T_{v}$ but also $I A T_{t_{0}}$ is impacted, giving researchers some indication of faking, which again points to the clear benefit of using diffusion-model-based IAT effects. Naïve faking did not impact $I A T_{t_{0}}$, a finding that again highlights the idea that naïve and informed faking are implemented by participants in different ways (see Röhner \& Ewers, 2016b; see also Röhner et al., 2013).

\section{Limitations}

Of course the current study is limited by the fact that we used only one IAT that measured the construct of extraversion. Thus, the results should be replicated with IATs that measure other constructs. In addition, we used only the EZ diffusion model here. Of course, other diffusion model tools have been proposed as well (e. g., HDDM, Wiecki et al., 2013; HDM, Vandekerckhove et al., 2011). However, these tools require more knowledge about diffusion models than EZ, which is quite accessible even to novices in diffusion modeling. Because our goal here was to investigate whether the easiest tool would help us better understand IAT results, we thus constrained ourselves to EZ.

\section{Summary and conclusion}

To summarize, the IAT is a very prominent measure (Bosson et al., 2000; Rudolph et al., 2008) that is often used by researchers to assess implicit associations and that is very popular among nonscientists because of its presence in the media (for an example, see Nosek, 2007). The latter seems problematic as faking becomes easier and more likely when test takers have greater knowledge about the test procedure (see, e. g., Dalen, Stanton, \& Roberts, 2001). It is thus no wonder that the fakeability of the IAT has been well-documented (e. g., Fiedler \& Bluemke, 2005; Röhner et al., 2011, 2013; Steffens, 2004) especially for the situation in which participants are informed about how the IAT works (i.e., informed faking; see, e. g., Röhner et al., 2011). The IAT is often used to assess socially stigmatized associations that might motivate people to fake (e. g., Agerström \& Rooth, 2011; Banse et al., 2010; Banse et al., 2001; Carlsson \& Björklund, 2010; Gray et al., 2005; Greenwald \& Banaji, 1995; Greenwald et al., 1998; Latu et al., 2011). Thus, faking is a serious problem not only because faking is possible and even likely but also because people are likely to have knowledge about how to fake. The $D$ measure includes different components such as the strength of implicit association but also the impact of faking that cannot be decomposed. The diffusion model, although admittedly much more complex than the $D$ measure, has been shown to represent one possible way to better comprehend the 
factors that are associated with the IAT effect (see Klauer et al., 2007; Röhner \& Ewers, 2016b).

What do our results mean for researchers who might be willing to (re-)analyze their IAT data with diffusion model analyses in applied settings (i.e., where faked data are not separated from nonfaked data by group assignment)? Does using $I A T_{v}$ instead of the $D$ measure automatically adjust for faking? No, it is not yet possible to use $I A T_{v}$ instead of the $D$ measure to produce an IAT measure that is automatically adjusted for faking. In replicating previous results that were obtained with the fast-dm tool (Röhner \& Ewers, 2016b), the present study demonstrated that faking has an impact on $I A T_{v}$. Does faking somehow stand out when diffusion-model-based IAT effects are used? Yes, with diffusion model analyses, some indications of faking are given. As was already shown for fast-dm (Röhner \& Ewers, 2016b), IAT might provide some indication of faking under informed faking conditions when computed with EZ. In addition to previous findings, EZ indicates an impact of informed faking on $I A T_{t_{0}}$. For example, unusually high or low values on $I A T_{\mathrm{a}}$ and $I A T_{t_{0}}$ may indicate that participants did not follow the standard instructions for the IAT. Is it advantageous to use diffusion model analyses? Yes, the present study underscores the idea that using diffusion model analyses to compute the diffusion-model-based IAT effects is quite helpful for getting a better idea about the components that comprise IAT results. Basic research in particular might profit from IAT effects for which researchers at least attempted to separate different sources of variance from each other. For example, if one wants to assess socially stigmatized associations with the IAT, it might be advantageous not only to compute the $D$ measure but also the diffusion-model-based IAT effects to get a better impression of the to-be-measured association, the speed-accuracy setting, and the processes that underlie faking.

With fast-dm, and as shown in this study also with EZ, there are at least two tools that researchers can use to analyze their IAT data in a more detailed manner, tools that even allow them to get some first indications of faking. Both tools yield quite comparable results, whereby EZ might be the easiest way and, under some conditions, also the more powerful.

\section{Authors' note}

Jessica Röhner, Department of Psychology, Chemnitz University of Technology, Germany; Philipp Thoss, Chemnitz, Germany. Correspondence concerning this article should be addressed to Jessica Röhner, Department of Psychology, Chemnitz University of Technology, D-09107 Chemnitz, Germany, Phone: +49 (0)371/ 531-38388, Fax: +49 (0)371/ 531-27459

\section{References}

Agerström, J. \& Rooth, D.-O. (2011). The role of automatic obesity stereotypes in real hiring discrimination. Journal of Applied Psychology, 96, 790-805. doi:10.1037/a0021594

Back, M. D., Schmukle, S. C., \& Egloff, B. (2005). Measuring task-switching ability in the Implicit Association Test. Experimental Psychology, 52, 167-179. doi:10.1027/ 1618-3169.52.3.167

Back, M. D., Schmukle, S. C., \& Egloff, B. (2009). Predicting actual behavior from the explicit and implicit selfconcept of personality. Journal of Personality and Social Psychology, 97, 533-548. doi:10.1037/a0016229

Banse, R., Schmidt, A. F., \& Clarbour, J. (2010). Indirect measures of sexual interest in child sex offenders: A multimethod approach. Criminal Justice and Behavior, 37, 319-335. doi:10.1177/0093854809357598

Banse, R., Seise, J., \& Zerbes, N. (2001). Implicit attitudes towards homosexuality: Reliability, validity, and controllability of the IAT. Zeitschrift für Experimentelle Psychologie, 48, 145-160. doi:10.1026//0949-3946.48. 2.145

Bar-Anan, Y. \& Nosek, B. A. (2014). A comparative investigation of seven indirect attitude measures. Behavior Research Methods, 46, 668-688. doi:10.3758/s13428013-0410-6

Bosson, J. K., Swann, W. B., Jr., \& Pennebaker, J. W. (2000). Stalking the perfect measure of implicit self-esteem: The blind men and the elephant revisited? Journal of Personality and Social Psychology, 79, 631-643. doi:10. 1037/0022-3514.79.4.631

Carlsson, R. \& Björklund, F. (2010). Implicit stereotype content: Mixed stereotypes can be measured with the Implicit Association Test. Social Psychology, 41, 213-222. doi:10.1027/1864-9335/a000029

Dalen, L. H., Stanton, N. A., \& Roberts, A. D. (2001). Faking personality questionnaires in personnel selection. Journal of Management Development, 20, 729742. doi:10.1108/02621710110401428

De Houwer, J., Beckers, T., \& Moors, A. (2007). Novel attitudes can be faked on the Implicit Association Test. Journal of Experimental Social Psychology, 43, 972978. doi:10.1016/j.jesp.2006.10.007

Faul, F., Erdfelder, E., Lang, A.-G., \& Buchner, A. (2007). G* power3: A flexible statistical power analysis program for the social, behavioral, and biomedical sciences. Behavior Research Methods, 39, 175-191. doi:10.3758/ BF03193146

Fiedler, K. \& Bluemke, M. (2005). Faking the IAT: Aided and unaided response control on the Implicit Association 
Tests. Basic and Applied Social Psychology, 27, 307316. doi:10.1207/s15324834basp2704_3

Gawronski, B. (2002). What does the Implicit Association Test measure? A test of the convergent and discriminant validity of prejudice-related IATs. Experimental Psychology, 49, 171-180. doi:10.1026//1618-3169.49.3. 171

Grasman, R. P., Wagenmakers, E.-J., \& van der Maas, H. L. (2009). On the mean and variance of response times under the diffusion model with an application to parameter estimation. Journal of Mathematical Psychology, 53, 55-68. doi:10.1016/j.jmp.20

Gray, N. S., Brown, A. S., MacCulloch, M. J., Smith, J., \& Snowden, R. J. (2005). An implicit test of the associations between children and sex in pedophiles. Journal of Abnormal Psychology, 114, 304-308. doi:10.1037/ 0021-843X.114.2.304

Greenwald, A. G. \& Banaji, M. R. (1995). Implicit social cognition: Attitudes, self-esteem, and stereotypes. Psychological Review, 102, 4-27. doi:10.1037/0033-295X. 102.1.4

Greenwald, A. G., McGhee, D., \& Schwartz, J. (1998). Measuring individual differences in implicit cognition: The Implicit Association Test. Journal of Personality and Social Psychology, 74, 1464-1480. doi:10.1037/ 0022-3514.74.6.1464

Greenwald, A. G., Nosek, B., \& Banaji, M. (2003a). Understanding and using the Implicit Association Test: I. An improved scoring algorithm. Journal of Personality and Social Psychology, 85, 197-216. doi:10.1037/00223514.85.2.197

Greenwald, A. G., Nosek, B., \& Banaji, M. (2003b). 'Understanding and using the Implicit Association Test: I. An improved scoring algorithm': Correction to Greenwald et al. (2003). Journal of Personality and Social Psychology, 85, 481. doi:10.1037/h0087889

Hofmann, W., Gawronski, B., Gschwendner, T., Le, H., \& Schmitt, M. (2005). A meta-analysis on the correlation between the Implicit Association Test and explicit self-report measures. Personality and Social Psychology Bulletin, 31, 1369-1385. doi:10 . 1177 / 0146167205275613

Klauer, K. C., Voss, A., Schmitz, F., \& Teige-Mocigemba, S. (2007). Process components of the Implicit Association Test: A diffusion-model analysis. Journal of Personality and Social Psychology, 93, 353-368. doi:10 . 1037/0022-3514.93.3.353

Latu, I. M., Stewart, T. L., Myers, A. C., Lisco, C. G., Estes, S. B., \& Donahue, D. K. (2011). What we "say" and what we "think" about female managers: Explicit versus implicit associations of women with success. Psy- chology of Women Quarterly, 35, 252-266. doi:10.1177/ 0361684310383811

McDaniel, M. J., Beier, M. E., Perkins, A. W., Goggin, S., \& Frankel, B. (2009). An assessment of the fakeability of self-report and implicit personality measures. Journal of Research in Personality, 43, 682-685. doi:10.1016/j. jrp.2009.01.011

McFarland, S. \& Crouch, Z. (2002). A cognitive skill confound on the Implicit Association Test. Social Cognition, 20, 483-510. doi:10.1521/soco.20.6.483.22977

Mierke, J. \& Klauer, K. C. (2003). Method-specific variance in the Implicit Association Test. Journal of Personality and Social Psychology, 85, 1180-1192. doi:10.1037/ 0022-3514.85.6.1180

Nosek, B. (2007). Implicit Association Test. [video file]. Retrieved from https://www. youtube. com $/$ watch ? $\mathrm{v}=$ n5Q5FQfXZag\&feature=related

Ratcliff, R. (1978). A theory of memory retrieval. Psychological Review, 85, 59-108. doi:10.1037/0033-295X.85.2.59

Ratcliff, R. (2008). The EZ diffusion method: Too EZ? Psychonomic Bulletin \& Review, 15, 1218-1228. doi:10 . 3758/PBR.15.6.1218

Ratcliff, R. (2014). Measuring psychometric functions with the diffusion model. Journal of Experimental Psychology: Human Perception and Performance, 40, 870-888. doi:10.1037/a0034954

Ratcliff, R., Gomez, P., \& McKoon, G. (2004). A diffusion model account of the lexical decision task. Psychological Review, 111, 159-182. doi:10.1037/0033-295X.111. 1.159

Ratcliff, R. \& Rouder, J. N. (1998). Modeling response times for two-choice decisions. Psychological Science, 9, 347-356. doi:10.1111/1467-9280.00067

Ratcliff, R. \& Rouder, J. N. (2000). A diffusion model account of masking in two-choice letter identification. Journal of Experimental Psychology: Human Perception and Performance, 26, 127-140. doi:10.1037/00961523.26.1.127

Ratcliff, R., Thapar, A., Gomez, P., \& McKoon, G. (2004). A diffusion model analysis of the effects of aging in the lexical-decision task. Psychology and Aging, 19, 278289. doi:10.1037/0882-7974.19.2.278

Ratcliff, R., Thapar, A., \& McKoon, G. (2001). The effects of aging on reaction time in a signal detection task. Psychology and Aging, 16, 323-341. doi:10.1037//0882 7974.16.2.323

Ratcliff, R., Thapar, A., \& McKoon, G. (2006). Aging and individual differences in rapid two-choice decisions. Psychonomic Bulletin \& Review, 13, 626-635. doi:10.3758/ BF03193973

Ratcliff, R. \& Tuerlinckx, F. (2002). Estimating parameters of the diffusion model: Approaches to dealing with

The Quantitative Methods for Psychology 
contaminant reaction times and parameter variability. Psychonomic Bulletin \& Review, 9, 438-481. doi:10. 3758/BF03196302

Röhner, J. \& Ewers, T. (2016a). How to analyze (faked) Implicit Association Test data by applying diffusion model analyses with the fast-dm software: A companion to Röhner \& Ewers (2016). The Quantitative Methods in Psychology, 12, 220-231. doi:10/20982/tqmp.12. 3.p220

Röhner, J. \& Ewers, T. (2016b). Trying to separate the wheat from the chaff: Construct- and faking-related variance on the Implicit Association Test (IAT). Behavior Research Methods, 48, 243-258. doi:10.3758/s13428015-0568-1

Röhner, J., Schröder-Abé, M., \& Schütz, A. (2011). Exaggeration is harder than understatement, but practice makes perfect! Faking success in the IAT. Experimental Psychology, 58, 464-472. doi:10.1027/1618-3169/ a000114

Röhner, J., Schröder-Abé, M., \& Schütz, A. (2013). What do fakers actually do to fake the IAT? An investigation of faking strategies under different faking conditions. Journal of Research in Personality, 47, 330-338. doi:10. 1016/j.jrp.2013.02.009

Rudolph, A., Schröder-Abé, M., Schütz, A., Gregg, A. P., \& Sedikides, C. (2008). Through a glass, less darkly? Reassessing convergent and discriminant validity in measures of implicit self-esteem. European Journal of Psychological Assessment, 24, 273-281. doi:10.1027/ 1015-5759.24.4.273

Schmitz, F. \& Voss, A. (2012). Decomposing task-switching costs with the diffusion model. Journal of Experimental Psychology: Human Perception and Performance, 38, 222-250. doi:10.1037/a0026003

Spaniol, J., Madden, D. J., \& Voss, A. (2006). A diffusion model analysis of adult age differences in episodic and semantic long-term memory retrieval. Journal of Experimental Psychology: Learning, Memory, and Cognition, 32, 101-117. doi:10.1037/0278-7393.32.1.101

Steffens, M. (2004). Is the Implicit Association Test immune to faking? Experimental Psychology, 51, 165179. doi:10.1027/1618-3169.51.3.165

Thapar, A., Ratcliff, R., \& McKoon, G. (2003). A diffusion model analysis of the effects of aging on letter discrimination. Psychology and Aging, 18, 415-429. doi:10.1037/0882-7974.18.3.415

Van Ravenzwaaij, D., Donkin, C., \& Vandekerckhove, J. (2016). The EZ diffusion model provides a powerful test of simple empirical effects. Psychonomic Bulletin \& Review, 24, 547-446. doi:10.3758/s13423-016-1081-y

Van Ravenzwaaij, D. \& Oberauer, K. (2009). How to use the diffusion model: Parameter recovery of three meth- ods: EZ, fast-dm, and DMAT. Journal of Mathematical Psychology, 53, 463-473. doi:10.1016/j.jmp.2009.09. 004

Vandekerckhove, J. \& Tuerlinckx, F. (2007). Fitting the Ratcliff diffusion model to experimental data. Psychonomic Bulletin \& Review, 14, 1011-1026. doi:10.3758/ BF03193087

Vandekerckhove, J. \& Tuerlinckx, F. (2008). Diffusion model analysis with Matlab: A DMAT primer. Behavior Research Methods, 40, 61-72. doi:10.3758/BRM.40. 1.61

Vandekerckhove, J., Tuerlinckx, F., \& Lee, M. D. (2011). Hierarchical diffusion models for two-choice response times. Psychological Methods, 16, 44-62. doi:10.1037/ a0021765

Voss, A., Nagler, M., \& Lerche, V. (2013). Diffusion models in experimental psychology: A practical introduction. Experimental Psychology, 60, 385-402. doi:10.1027/ 1618-3169/a000218

Voss, A., Rothermund, K., \& Brandstädter, J. (2008). Interpreting ambiguous stimuli: Separating perceptual and judgmental biases. Journal of Experimental Social Psychology, 44, 1048-1056. doi:10.1016/j.jesp.2007.10. 009

Voss, A., Rothermund, K., Gast, A., \& Wentura, D. (2013). Cognitive processes in categorical and associative priming: A diffusion model analysis. Journal of EXperimental Psychology: General, 142, 536-559. doi:10. 1037/a0029459

Voss, A., Rothermund, K., \& Voss, J. (2004). Interpreting the parameters of the diffusion model: An empirical validation. Memory \& Cognition, 32, 1206-1220. doi:10. 3758/BF03196893

Voss, A. \& Voss, J. (2007). Fast-dm: A free program for efficient diffusion model analysis. Behavior Research Methods, 39, 767-775. doi:10.3758/BF03192967

Voss, A. \& Voss, J. (2008). A fast numerical algorithm for the estimation of diffusion model parameters. Journal of Mathematical Psychology, 52, 1-9. doi:10.1016/j.jmp. 2007.09.005

Voss, A., Voss, J., \& Lerche, V. (2015). Assessing cognitive processes with diffusion model analyses: A tutorial based on fast-dm-30. Frontiers in Psychology, 6, 336339. doi:10.3389/fpsyg.2015.00336

Wagenmakers, E. J., van der Maas, H. L. J., Dolan, C. V., \& Grasman, R. P. (2008). EZ does it! Extensions of the EZdiffusion model. Psychonomic Bulletin \& Review, 15, 1229-1235. doi:10.3758/PBR.15.6.1229

Wagenmakers, E. J., van der Maas, H. L., \& Grasman, R. P. (2007). An EZ-diffusion model for response time and accuracy. Psychonomic Bulletin \& Review, 14, 3-22. doi:10.3758/BF03194023

The Quantitative Methods for Psychology 
Wiecki, T. V., Sofer, I., \& Frank, M. J. (2013). HDDM: Hierarchical Bayesian estimation of the drift-diffusion model in python. Frontiers in Neuroinformatics, 7, 1429. doi:10.3389/fninf.2013.00014

\section{Citation}

Röhner, J. \& Thoss, P. (2018). EZ: An easy way to conduct a more fine-grained analysis of faked and nonfaked Implicit Association Test (IAT) data. The Quantitative Methods for Psychology, 14(1), 17-37. doi:10.20982/tqmp.14.1.p017

Copyright (C) 2018, Röhner and Thoss. This is an open-access article distributed under the terms of the Creative Commons Attribution License (CC BY). The use, distribution or reproduction in other forums is permitted, provided the original author(s) or licensor are credited and that the original publication in this journal is cited, in accordance with accepted academic practice. No use, distribution or reproduction is permitted which does not comply with these terms.

Tables 2 to 5 follow. 


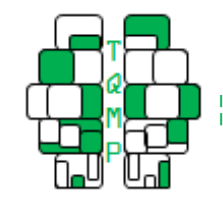

2018 - Vol. 14 - no. 1

ב

政

हृ.

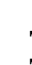

.



要

承

永

$\stackrel{9}{\Xi}$

$\$$

ç

䒕

荬

要

(

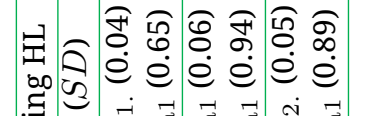
要

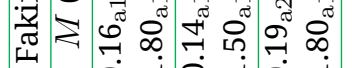
क्व

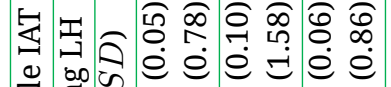

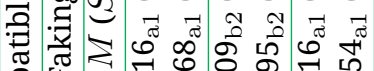

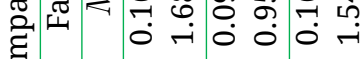
苛

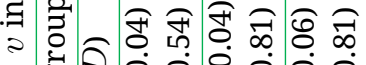

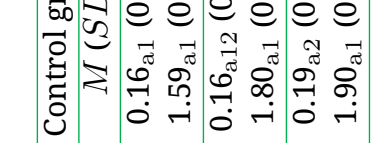

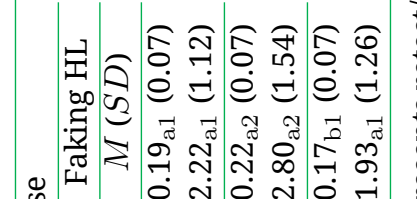
弯

년응

प्र

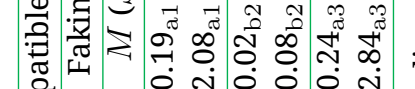
: ว

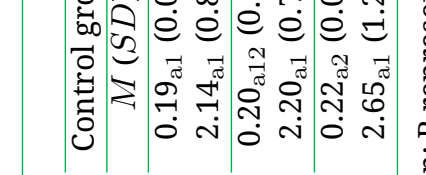

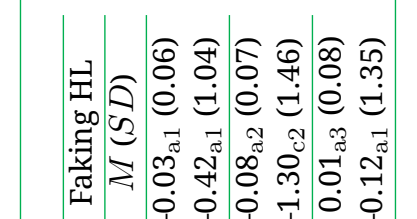

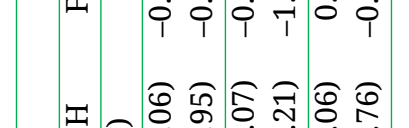

2

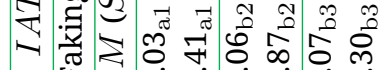

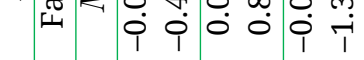

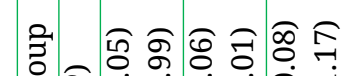

品 $0 \dot{0} \dot{0}$

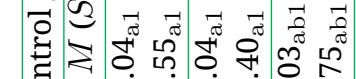

ठै।

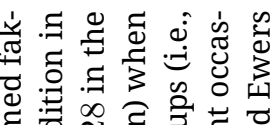
CrossMark

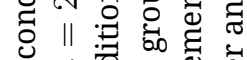

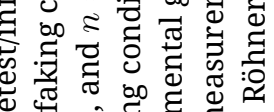

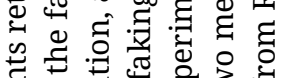

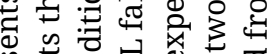

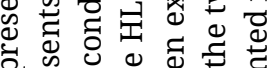
s.

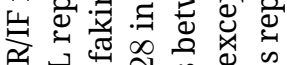
60․

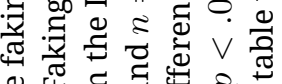
㟧

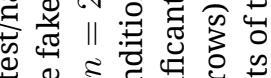
政 क के an क्षे 吨 品 踏

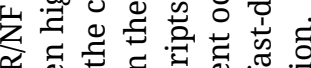

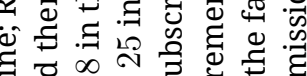

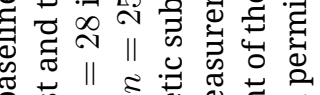

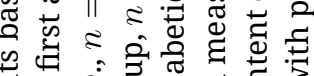
可

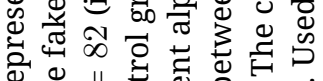

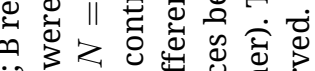

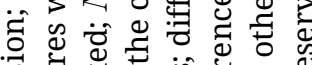
ơ

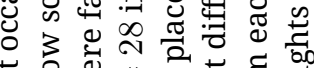
풍워 岛

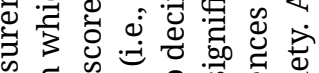

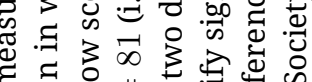

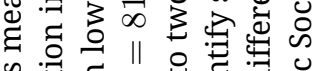

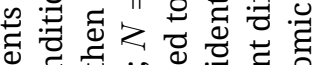

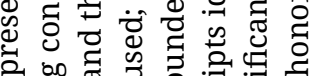
훙

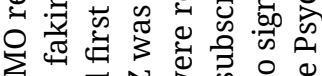
讨

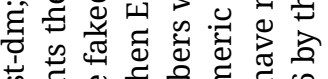

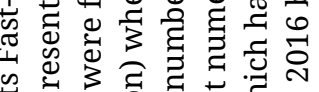

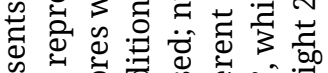

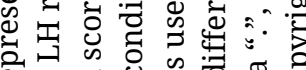

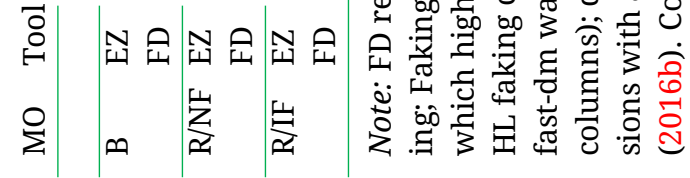
क्ष 


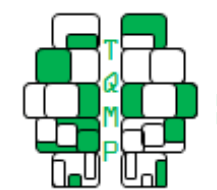

2018 - Vol. 14 - no. 1

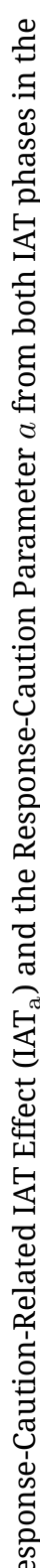

急

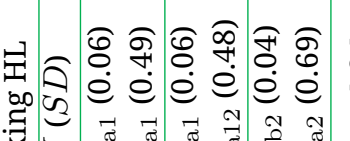

蛋 艺

政

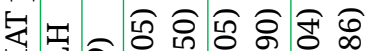

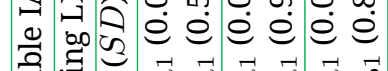

表表

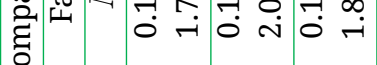

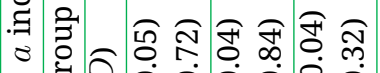

क्ष

苟 娄

A

1.

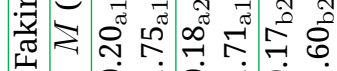

党

2 न न

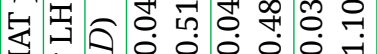

0.500

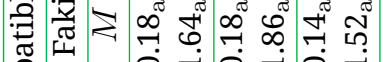

ఫั

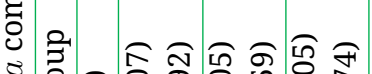

.

क. Â

(2)

है

ণัن

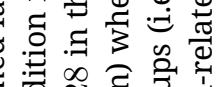

CrossMark

tcilckfor updates

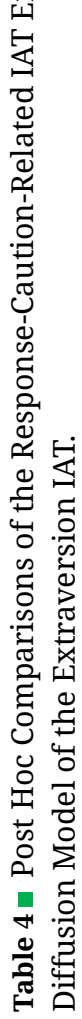

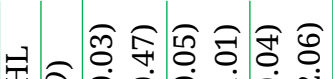

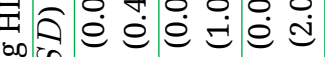

菏

疋

- $0^{\circ}$ i

ม

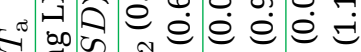

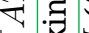

m.

娄

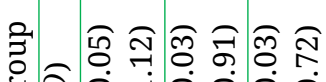

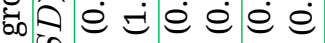

:

ชे

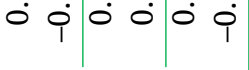

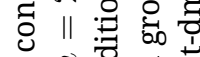

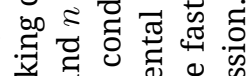

\%

东芯

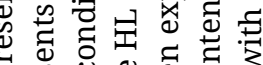

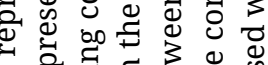

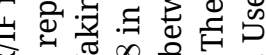

60․

政 $\vee$

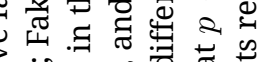

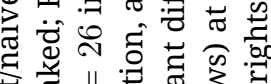

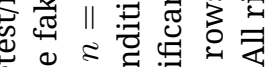

क्ष क्षे है है

उ 0 bo क्ष

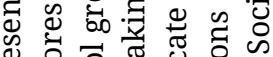

尊

눙 동

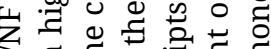

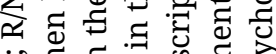

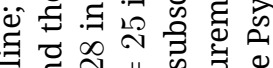

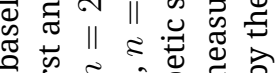

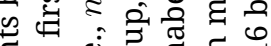

唍

要

$\infty$ उ

के के

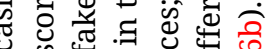

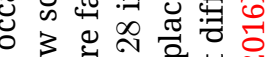

范

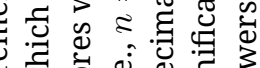

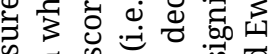

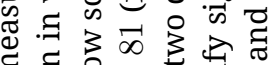

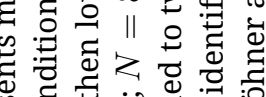

记

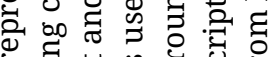

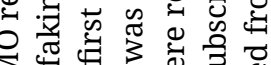

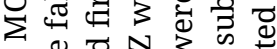

है

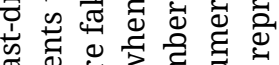

品过

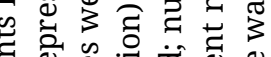

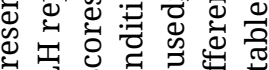

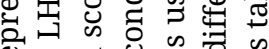

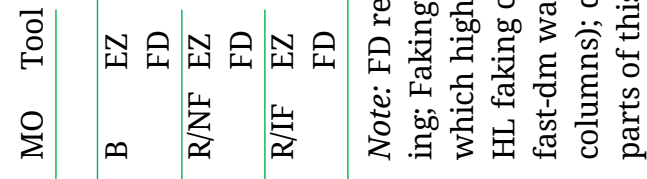




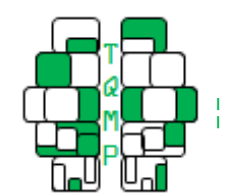

2018 - Vol. 14 no. 1

足

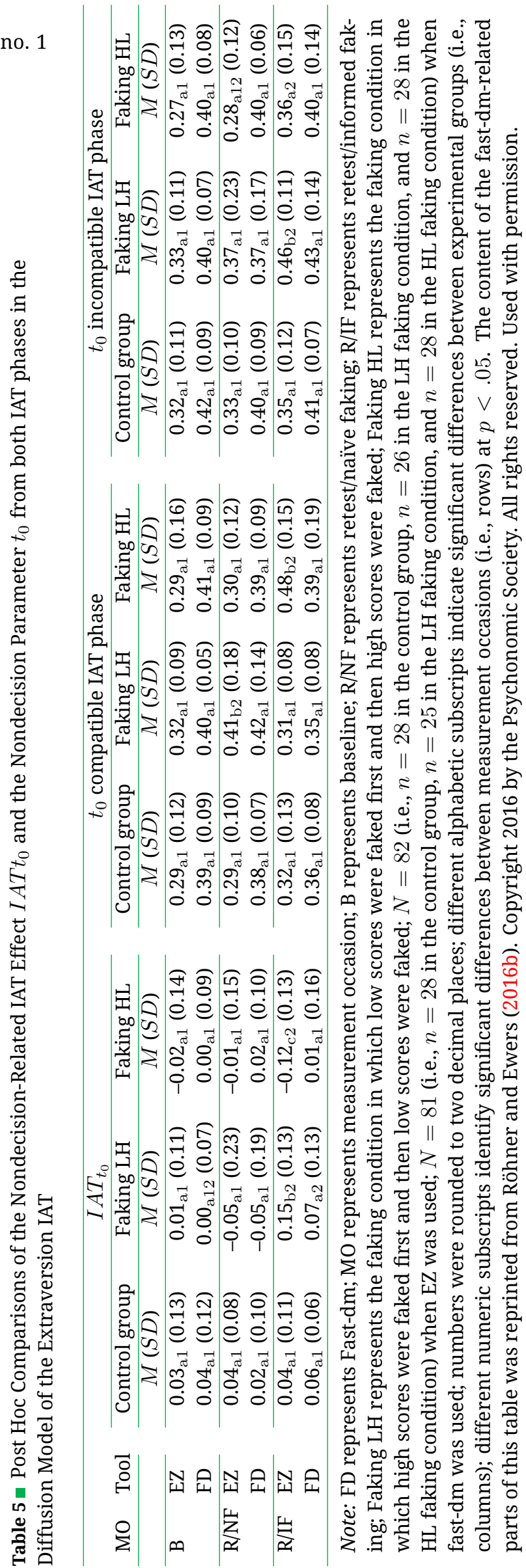

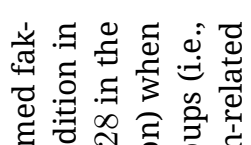

